\title{
An Optimized Stochastic Techniques related to Option Pricing
}

\author{
Venelin Todorov ${ }^{* \dagger}$, Ivan Dimov ${ }^{\dagger}$, Stefka Fidanova ${ }^{\dagger}$, Stoyan Apostolov ${ }^{\ddagger}$ \\ *Institute of Mathematics and Informatics \\ Bulgarian Academy of Sciences \\ 8 Acad. G. Bonchev Str., 1113 Sofia, Bulgaria \\ ${ }^{\dagger}$ Institute of Information and Communication Technologies \\ Bulgarian Academy of Sciences \\ 25A Acad. G. Bonchev Str., 1113 Sofia, Bulgaria \\ ${ }^{\ddagger}$ Faculty of Mathematics and Informatics, Sofia University, Sofia 1126, Bulgaria
}

Email: vtodorov@math.bas.bg, venelin@ parallel.bas.bg, ivdimov@bas.bg, stefka@ parallel.bas.bg, stoyanrapostolov@gmail.com

\begin{abstract}
Recently stochastic methods have become very important tool for high performance computing of very high dimensional problems in computational finance. The advantages and disadvantages of the different highly efficient stochastic methods for multidimensional integrals related to evaluation of European style options will be analyzed. Multidimensional integrals up to 100 dimensions related to European options will be computed with highly efficient optimized lattice rules.
\end{abstract}

\section{INTRODUCTION}

$\mathbf{R}$ ECENTLY Monte Carlo (MC) and quasi-Monte Carlo (QMC) approaches have become a very attractive and necessary computational tools in finance [8]. The field of computational finance is becoming more complicated with increasing number of applications [2], [3]. The pricing of options is a very important in financial markets today and especially difficult when the dimension of the problem goes higher [2], [8], [9], [12]. MC and QMC methods are appropriate for solving multidimensional problems [4], since their computational complexity increases polynomially, but not exponentially with the dimensionality. MC methods are used not only for option pricing, but also in other problems in computational finance. The basic definitions and terminology used in the paper can be found in [1], [8].

The paper is organized as follows. The problem setting and motivation is presented in Section II. Some basic notations about the stochastic methods that we are going to use are presented in III. Numerical study and discussions are given in Section IV. The conclusions are given in Section V.

Venelin Todorov is supported by the National Scientific Program "Information and Communication Technologies for a Single Digital Market in Science, Education and Security (ICT in SES)", contract No DO1-205/23.11.2018, financed by the Ministry of Education and Science in Bulgaria and by the Bulgarian National Science Fund under Project DN 12/5-2017 "Efficient Stochastic Methods and Algorithms for Large-Scale Problems". Stoyan Apostolov is supported by the Bulgarian National Science Fund under Project KP06-M32/2 - 17.12.2019 "Advanced Stochastic and Deterministic Approaches for Large-Scale Problems of Computational Mathematics". The work is also supported by the Project KP-06-Russia/17 "New Highly Efficient Stochastic Simulation Methods and Applications" funded by the National Science Fund - Bulgaria.

\section{Problem SEtTings AND Motivation}

Consider a European call option [8] whose payoff depends on $k>1$ assets with prices $S_{i}, i=1, \ldots, k$. The payoff is the act or occasion of receiving money or material gain especially as compensation or as a bribe. Following [8] we assume that at expiry time $T$, and risk-free interest rate $r$, the payoff is given by $h\left(S_{1}^{\prime}, \ldots, S_{k}^{\prime}\right)$, where $S^{\prime}$ denotes the value of the $i$-th asset at expiry. Then the value of the option satisfies:

$$
\begin{gathered}
V=e^{-r(T-t)}(2 \pi(T-t))^{-k / 2}(\operatorname{det} \Sigma)^{-1 / 2}\left(\sigma_{1} \ldots \sigma_{k}\right)^{-1} \\
\int_{0}^{\infty} \ldots \int_{0}^{\infty} \frac{h\left(S_{1}^{\prime}, \ldots, S_{k}^{\prime}\right)}{S_{1}^{\prime} \ldots S_{k}^{\prime}} \\
\exp \left(-0.5 \alpha^{\top} \Sigma^{-1} \alpha\right) \mathrm{d} S_{1}^{\prime} \ldots \mathrm{d} S_{k}^{\prime}, \\
\alpha_{i}=\left(\sigma_{i}(T-t)^{1 / 2}\right)^{-1}\left(\ln \left(S_{i}^{\prime} / S_{i}\right)-\left(r-\sigma_{i}^{2} / 2\right)(T-t)\right) .
\end{gathered}
$$

According to [8] the most important case in recent models is when the payoff function is the exponent function.

We will now give a brief explanation which demonstrates the strength of the MC and QMC approach. This is a case of practical high performance computations showing the high power and efficiency of the stochastic approach versus the deterministic one [4]. According to [4] we will choose 100 nodes on the each of the coordinate axes in the $s$-dimensional cube $G=E^{s}$ and we have to evaluate about $10^{100}$ values of the function $f(x)$. Assume a time of $10^{-7} s$ is necessary for calculating one value of the function [4]. So, a time of order $10^{93} \mathrm{~s}$ will be necessary for computation of the integral, and 1 year has $31536 \times 10^{3} \mathrm{~s}$.

Now MC approach [4] consists of generating $\mathrm{N}$ pseudo random values (points) (PRV) in $G$; in evaluating the values of $f(x)$ at these points; and averaging the computed values of the function. For each uniformly distributed random (UDR) point in $G$ we have to generate 100 UDR numbers in $[0,1]$. The probable error is estimated in [4]:

$$
N \approx\left(\frac{0.6745\|f\|_{L_{2}}}{c M}\right)^{2} \times h^{-6}
$$


Assume that the expression in front of $h^{-6}$ is of order 1 [4]. Here $h=0.1$, and we have $N \approx 10^{6}$; so, it will be necessary to generate $100 \times 10^{6}=10 \times 10^{7} \mathrm{PRV}$. Usually, 2 operations are sufficient to generate a single PRV. According to [4] the time required to generate one PRV is the same as that for computation the value of $f(x)$. So, in order to solve the task with the same accuracy, a time of

$$
10 \times 10^{7} \times 2 \times 10^{-7} \approx 20 s
$$

will be necessary. We summarize that in the case of 100dimensional integral it is $5 \times 10^{91}$ times faster than the deterministic one. Also the stochastic approach is more accurate than the deterministic approach for higher dimensions. That motivates our study on the new highly efficient stochastic approaches for the problem under consideration.

\section{HIGHLY EFFICIENT STOCHASTIC APPROACHES}

We will make a brief description of the stochastic approaches that we are going to use in our survey. Such comparison, up to 100 dimensions, has been made for the first time for the problem under consideration.

\section{A. Lattice rules}

Fot the lattice point sets, please see cite[13], and more information can be found in the works of Sloan and Kachoyan [10], Sloan and Joe [11] and Hua and Wang [6].

We will use this rank-1 lattice sequence [13]:

$$
\mathbf{x}_{k}=\left\{\frac{k}{N} \mathbf{z}\right\}, k=1, \ldots, N,
$$

where $N$ is an integer, $N \geq 2, \mathbf{z}=\left(z_{1}, z_{2}, \ldots z_{s}\right)$ is the generating vector and $\{z\}$ denotes the fractional part of $z$. For the definition of the $E_{s}^{\alpha}(c)$ and $P_{\alpha}(z, N)$ see [13].

The existence of lattice point sets with low discrepancy and low worst case error are closely connected [13].

While the theoretical result establish the existence of optimal generating vectors the main bottleneck lies in the creation of the optimal vectors, especially for very high dimensions [8].

The first generating vector in our study is the generalized Fibonacci numbers of the corresponding dimension:

$$
\mathbf{z}=\left(1, F_{n}^{(s)}(2), \ldots, F_{n}^{(s)}(s)\right) .
$$

where we use that

$$
F_{n}^{(s)}(j):=F_{n+j-1}^{(s)}-\sum_{i=0}^{j-2} F_{n+i}^{(s)}
$$

and $F_{n+l}^{(s)}(l=0, \ldots, j-1, j$ is an integer, $2 \leq j \leq s)$ is the term of the $s$-dimensional Fibonacci sequence [13].

Then each component of the generating vector $\mathbf{z}$ is defined by a sum of some terms of the generalized Fibonacci sequence with dimensionality $s$. For example:

$$
\begin{gathered}
F_{n}^{(s)}(2)=F_{n+1}^{(s)}-F_{n}^{(s)}=\left(F_{n}^{(s)}+F_{n-1}^{(s)}+\ldots+\right. \\
\left.F_{n-s+1}^{(s)}\right)-F_{n}^{(s)}=F_{n-1}^{(s)}+\ldots+F_{n-s+1}^{(s)} .
\end{gathered}
$$

Our generating vector (3) is transformed into [6], [13]:

$\mathbf{z}=\left(1, F_{n-1}^{(s)}+F_{n-2}^{(s)}+\ldots+F_{n-s+1}^{(s)}, \ldots, F_{n-1}^{(s)}+F_{n-2}^{(s)}, F_{n-1}^{(s)}\right)$.

If we change the generating vector to be optimal in the way described in [7] we have improved the lattice sequence. This is a 200-dimensional base-2 generating vector of prime numbers for up to $2^{20}=1048576$ points, constructed recently by Dirk Nuyens [7]. The special choice of this optimal generating vector is definitely more efficient than the Fibonacci generating vector, which is only optimal for the two dimensional case [13]. For this improved lattice rule, presented in the paper, is satisfied [7]:

$$
D_{N}^{*}=\mathcal{O}\left(\frac{\log ^{s} N}{N}\right)
$$

\section{NumERICAL EXAMPLES AND RESUlTS}

The numerical study includes high performance computing of the multidimensional integrals

$$
I_{s}=\int_{[0,1]^{s}} \exp \left(\prod_{i=1}^{s} x_{i}\right)
$$

We will use the expansion of the exponential function in Taylor series and integrating $\left(x_{1} \cdots x_{s}\right)^{n}$ :

$$
\begin{gathered}
\int_{[0,1]^{s}} \exp \left(\prod_{i=1}^{s} x_{i}\right)= \\
=\sum_{n=0}^{\infty} \frac{1}{(n+1)^{s} n !}={ }_{s} F_{s}(1, \cdots, 1 ; 2, \cdots, 2 ; 1),
\end{gathered}
$$

where ${ }_{p} F_{q}\left(a_{1}, \cdots, a_{p} ; b_{1}, \cdots, b_{q} ; x\right)$ is the generalized hypergeometric function

$$
{ }_{p} F_{q}\left(a_{1}, \cdots, a_{p} ; b_{1}, \cdots, b_{q} ; x\right)=\sum_{n=0}^{\infty} \frac{\left(a_{1}\right)_{n} \cdots\left(a_{p}\right)_{n}}{\left(b_{1}\right)_{n} \cdots\left(b_{q}\right)_{n}} \frac{x^{n}}{n !},
$$

and $(c)_{n}=c(c+1) \cdots(c+n-1)$ is the Pochhammer symbol.

$$
\int_{[0,1]^{3}} \exp \left(x_{1} x_{2} x_{3}\right) \approx 1.14649907 .
$$

$$
\int_{[0,1]^{5}} \exp \left(\sum_{i=1}^{5} 0.5 a_{i} x_{i}^{2}\left(2+\sin \sum_{j=1, j \neq i}^{5} x_{j}\right)\right) \approx 2.923651
$$

where $a_{i}=(1,0.5,0.2,0.2,0.2)$.

$$
\int_{[0,1]^{8}} \exp \left(\sum_{i=1}^{8} 0.1 x_{i}\right)=1.496805
$$

$$
\int_{[0,1]^{20}} \exp \left(\prod_{i=1}^{20} x_{i}\right) \approx 1.00000949634
$$


We also have done high performance computing with our methods for the first time on a 100 dimensional integral:

$$
I_{100}=\int_{[0,1]^{100}} \exp \left(\prod_{i=1}^{100} x_{i}\right) \text {. }
$$

We calculate his reference value by using the exponential function in Taylor series and integrating $\left(x_{1} \cdots x_{100}\right)^{n}$ we receive

$$
\begin{gathered}
\int_{[0,1]^{100}} \exp \left(\prod_{i=1}^{100} x_{i}\right)= \\
=\sum_{n=0}^{\infty} \frac{1}{(n+1)^{100} n !}={ }_{100} F_{100}(1, \cdots, 1 ; 2, \cdots, 2 ; 1) .
\end{gathered}
$$

We also include in the experiments the 50-dimensional integral of the same kind:

$$
I_{50}=\int_{[0,1]^{50}} \exp \left(\prod_{i=1}^{50} x_{i}\right) .
$$

The results are given in the Tables including the relative error (RE) of the MC and QMC method that has been used, the CPU-time (T) in seconds and the number of realizations of the random variable (\#). We will make a high performance computation, including the Optimized lattice rule (OP), the Fibonacci based rule (FI), the Adaptive approach (AD) and the Sobol quasi-random sequence (SO).

Table I

ALGORITHMIC COMPARISON OF RE FOR (7)

\begin{tabular}{|c|c|c|c|c|c|c|c|c|}
\hline$\#$ & OP & T & AD & T & FI & T & SO & T \\
\hline 19513 & $1.93 \mathrm{e}-5$ & 0.01 & $3.21 \mathrm{e}-4$ & 2.21 & $4.69 \mathrm{e}-4$ & 0.02 & $4.98 \mathrm{e}-5$ & 0.56 \\
35890 & $3.18 \mathrm{e}-6$ & 0.04 & $6.55 \mathrm{e}-5$ & 6.41 & $5.46 \mathrm{e}-6$ & 0.06 & $1.56 \mathrm{e}-5$ & 1.45 \\
66012 & $2.65 \mathrm{e}-6$ & 0.07 & $5.12 \mathrm{e}-5$ & 9.86 & $5.34 \mathrm{e}-6$ & 0.11 & $8.11 \mathrm{e}-6$ & 2.31 \\
121415 & $9.16 \mathrm{e}-7$ & 0.12 & $5.11 \mathrm{e}-5$ & 15.4 & $5.34 \mathrm{e}-6$ & 0.12 & $3.08 \mathrm{e}-6$ & 3.80 \\
223317 & $8.01 \mathrm{e}-7$ & 0.20 & $9.34 \mathrm{e}-5$ & 24.2 & $1.73 \mathrm{e}-6$ & 0.22 & $2.05 \mathrm{e}-6$ & 6.13 \\
\hline
\end{tabular}

Table II

ALGORITHMIC COMPARISON OF RE FOR THE (7)

\begin{tabular}{|c|c|c|c|c|}
\hline $\mathrm{T}$ & OP & AD & FI & SO \\
\hline 0.1 & $9.16 \mathrm{e}-7$ & $8.67 \mathrm{e}-4$ & $1.32 \mathrm{e}-6$ & $3.21 \mathrm{e}-4$ \\
1 & $6.37 \mathrm{e}-7$ & $2.96 \mathrm{e}-5$ & $3.22 \mathrm{e}-7$ & $8.21 \mathrm{e}-5$ \\
2 & $4.22 \mathrm{e}-7$ & $5.45 \mathrm{e}-4$ & $2.06 \mathrm{e}-7$ & $2.96 \mathrm{e}-5$ \\
5 & $1.84 \mathrm{e}-7$ & $1.14 \mathrm{e}-4$ & $1.47 \mathrm{e}-7$ & $5.00 \mathrm{e}-6$ \\
10 & $6.09 \mathrm{e}-8$ & $6.56 \mathrm{e}-5$ & $3.89 \mathrm{e}-7$ & $2.71 \mathrm{e}-6$ \\
20 & $1.57 \mathrm{e}-8$ & $2.04 \mathrm{e}-5$ & $1.53 \mathrm{e}-8$ & $1.65 \mathrm{e}-6$ \\
\hline
\end{tabular}

Table III

ALGORITHMIC COMPARISON OF RE FOR THE (8)

\begin{tabular}{|c|c|c|c|c|c|c|c|c|}
\hline$\#$ & OP & T & AD & T & FI & T & SO & T \\
\hline 13624 & $6.72 \mathrm{e}-5$ & 0.02 & $1.89 \mathrm{e}-3$ & 2.33 & $9.59 \mathrm{e}-4$ & 0.03 & $1.76 \mathrm{e}-4$ & 0.56 \\
52656 & $1.53 \mathrm{e}-5$ & 0.06 & $2.31 \mathrm{e}-3$ & 6.18 & $6.96 \mathrm{e}-4$ & 0.06 & $5.05 \mathrm{e}-5$ & 1.45 \\
103519 & $8.48 \mathrm{e}-6$ & 0.09 & $2.01 \mathrm{e}-3$ & 9.94 & $8.72 \mathrm{e}-5$ & 0.13 & $2.70 \mathrm{e}-5$ & 2.52 \\
203513 & $6.25 \mathrm{e}-6$ & 0.15 & $3.42 \mathrm{e}-4$ & 16.2 & $8.04 \mathrm{e}-5$ & 0.25 & $7.57 \mathrm{e}-6$ & 6.07 \\
400096 & $8.16 \mathrm{e}-7$ & 0.40 & $9.12 \mathrm{e}-4$ & 45.6 & $7.26 \mathrm{e}-5$ & 0.50 & $2.52 \mathrm{e}-6$ & 10.63 \\
\hline
\end{tabular}

Table IV

ALGORITHMIC COMPARISON OF RE FOR THE (8)

\begin{tabular}{|c|c|c|c|c|}
\hline $\mathrm{T}$ & OP & AD & FI & SO \\
\hline 0.1 & $3.07 \mathrm{e}-6$ & $1.34 \mathrm{e}-2$ & $7.26 \mathrm{e}-5$ & $8.22 \mathrm{e}-4$ \\
1 & $1.32 \mathrm{e}-6$ & $2.44 \mathrm{e}-3$ & $2.28 \mathrm{e}-5$ & $2.91 \mathrm{e}-4$ \\
5 & $1.13 \mathrm{e}-6$ & $4.93 \mathrm{e}-4$ & $5.94 \mathrm{e}-6$ & $1.71 \mathrm{e}-5$ \\
10 & $5.47 \mathrm{e}-7$ & $1.88 \mathrm{e}-3$ & $3.85 \mathrm{e}-7$ & $1.79 \mathrm{e}-5$ \\
20 & $3.52 \mathrm{e}-7$ & $2.71 \mathrm{e}-4$ & $7.49 \mathrm{e}-7$ & $4.71 \mathrm{e}-6$ \\
\hline
\end{tabular}

Table V

ALGORITHMiC COMPARISON OF RE FOR THE (9)

\begin{tabular}{|c|c|c|c|c|c|c|c|c|}
\hline$\#$ & OP & T & AD & T & FI & T & SO & T \\
\hline 16128 & $1.79 \mathrm{e}-6$ & 0.04 & $1.10 \mathrm{e}-5$ & 12.6 & $8.08 \mathrm{e}-4$ & 0.03 & $8.87 \mathrm{e}-5$ & 0.13 \\
32192 & $1.56 \mathrm{e}-6$ & 0.05 & $3.32 \mathrm{e}-5$ & 33.3 & $1.03 \mathrm{e}-4$ & 0.07 & $5.42 \mathrm{e}-5$ & 0.58 \\
64256 & $8.01 \mathrm{e}-7$ & 0.08 & $4.65 \mathrm{e}-5$ & 54.2 & $5.03 \mathrm{e}-5$ & 0.11 & $2.34 \mathrm{e}-5$ & 2.49 \\
128257 & $6.22 \mathrm{e}-7$ & 0.13 & $8.25 \mathrm{e}-6$ & 88.3 & $8.13 \mathrm{e}-6$ & 0.14 & $4.45 \mathrm{e}-6$ & 6.36 \\
510994 & $3.21 \mathrm{e}-7$ & 0.34 & $7.07 \mathrm{e}-6$ & 233.6 & $5.95 \mathrm{e}-6$ & 0.57 & $3.32 \mathrm{e}-6$ & 19.45 \\
\hline
\end{tabular}

Table VI

ALGORITHMIC COMPARISON OF RE FOR THE (9)

\begin{tabular}{|c|c|c|c|c|}
\hline $\mathrm{T}$ & OP & AD & FI & SO \\
\hline 1 & $2.18 \mathrm{e}-7$ & $6.34 \mathrm{e}-4$ & $5.34 \mathrm{e}-6$ & $2.02 \mathrm{e}-5$ \\
2 & $1.32 \mathrm{e}-7$ & $1.58 \mathrm{e}-4$ & $2.57 \mathrm{e}-6$ & $2.73 \mathrm{e}-5$ \\
5 & $9.03 \mathrm{e}-8$ & $1.44 \mathrm{e}-4$ & $1.52 \mathrm{e}-7$ & $8.88 \mathrm{e}-6$ \\
10 & $5.00 \mathrm{e}-8$ & $6.61 \mathrm{e}-5$ & $3.45 \mathrm{e}-6$ & $5.23 \mathrm{e}-6$ \\
20 & $2.55 \mathrm{e}-8$ & $2.77 \mathrm{e}-5$ & $1.82 \mathrm{e}-7$ & $2.11 \mathrm{e}-6$ \\
\hline
\end{tabular}

Table VII

ALGORITHMIC COMPARISON OF RE FOR THE (10)

\begin{tabular}{|c|c|c|c|c|c|c|c|c|}
\hline$\#$ & OP & T & AD & T & FI & T & SO & T \\
\hline 2048 & $2.84 \mathrm{e}-6$ & 0.02 & $1.14 \mathrm{e}-2$ & 8.6 & $8.22 \mathrm{e}-5$ & 0.03 & $8.44 \mathrm{e}-4$ & 0.13 \\
16384 & $1.04 \mathrm{e}-6$ & 0.12 & $4.96 \mathrm{e}-4$ & 60.3 & $3.12 \mathrm{e}-5$ & 0.13 & $6.82 \mathrm{e}-5$ & 1.68 \\
65536 & $9.21 \mathrm{e}-7$ & 0.91 & $9.75 \mathrm{e}-4$ & 474.2 & $1.36 \mathrm{e}-5$ & 1.17 & $8.34 \mathrm{e}-6$ & 8.69 \\
131072 & $6.15 \mathrm{e}-7$ & 2.13 & $1.25 \mathrm{e}-5$ & 888.3 & $8.85 \mathrm{e}-6$ & 2.34 & $3.77 \mathrm{e}-6$ & 14.36 \\
524288 & $5.33 \mathrm{e}-8$ & 8.13 & $1.96 \mathrm{e}-6$ & 2356 & $2.15 \mathrm{e}-6$ & 8.34 & $1.91 \mathrm{e}-7$ & 57 \\
\hline
\end{tabular}

Table VIII

ALGORITHMIC COMPARISON OF RE FOR THE (10)

\begin{tabular}{|c|c|c|c|c|}
\hline $\mathrm{T}$ & OP & AD & FI & SO \\
\hline 1 & $9.14 \mathrm{e}-7$ & $1.58 \mathrm{e}-3$ & $1.48 \mathrm{e}-5$ & $3.25 \mathrm{e}-5$ \\
2 & $1.08 \mathrm{e}-7$ & $1.028 \mathrm{e}-3$ & $9.17 \mathrm{e}-6$ & $3.97 \mathrm{e}-5$ \\
5 & $5.87 \mathrm{e}-8$ & $8.58 \mathrm{e}-4$ & $5.19 \mathrm{e}-6$ & $1.45 \mathrm{e}-5$ \\
10 & $3.56 \mathrm{e}-8$ & $4.31 \mathrm{e}-4$ & $1.73 \mathrm{e}-6$ & $2.71 \mathrm{e}-6$ \\
20 & $1.23 \mathrm{e}-8$ & $1.27 \mathrm{e}-4$ & $1.38 \mathrm{e}-7$ & $1.76 \mathrm{e}-6$ \\
\hline
\end{tabular}

Table IX

ALGORITHMIC COMPARISON OF RE FOR THE (12)

\begin{tabular}{|c|c|c|c|c|c|c|}
\hline$\#$ & OP & T & FI & T & SO & T \\
\hline $2^{10}$ & $7.88 \mathrm{e}-6$ & 0.05 & $6.23 \mathrm{e}-4$ & 0.08 & $8.88 \mathrm{e}-5$ & 3.5 \\
$2^{12}$ & $1.88 \mathrm{e}-6$ & 0.17 & $1.55 \mathrm{e}-4$ & 0.35 & $5.21 \mathrm{e}-5$ & 16 \\
$2^{16}$ & $8.44 \mathrm{e}-8$ & 2.14 & $9.72 \mathrm{e}-5$ & 5.21 & $9.11 \mathrm{e}-4$ & 73 \\
$2^{20}$ & $4.28 \mathrm{e}-8$ & 17.65 & $6.08 \mathrm{e}-5$ & 32.76 & $4.88 \mathrm{e}-6$ & 276 \\
\hline
\end{tabular}

For the 3-dimensional integral, for the number of samples 
Table X

ALGORITHMIC COMPARISON OF RE FOR THE (12)

\begin{tabular}{|c|c|c|c|}
\hline $\mathrm{T}$ & OP & FI & SO \\
\hline 1 & $9.14 \mathrm{e}-7$ & $1.58 \mathrm{e}-3$ & $1.48 \mathrm{e}-4$ \\
2 & $7.51 \mathrm{e}-7$ & $1.028 \mathrm{e}-3$ & $9.17 \mathrm{e}-5$ \\
10 & $9.34 \mathrm{e}-8$ & $3.01 \mathrm{e}-4$ & $8.73 \mathrm{e}-5$ \\
100 & $1.34 \mathrm{e}-9$ & $5.23 \mathrm{e}-5$ & $1.03 \mathrm{e}-5$ \\
\hline
\end{tabular}

Table XI

ALGORITHM COMPARISON OF THE RE FOR THE (11)

\begin{tabular}{|c|c|c|c|c|c|c|}
\hline$\#$ & OP & T & FI & T & SO & T \\
\hline $2^{10}$ & $6.83 \mathrm{e}-3$ & 0.05 & $4.13 \mathrm{e}-1$ & 0.06 & $6.31 \mathrm{e}-2$ & 18 \\
$2^{12}$ & $3.77 \mathrm{e}-4$ & 0.17 & $1.15 \mathrm{e}-1$ & 0.18 & $1.23 \mathrm{e}-2$ & 34 \\
$2^{16}$ & $3.36 \mathrm{e}-5$ & 9.1 & $6.12 \mathrm{e}-2$ & 9.2 & $2.31 \mathrm{e}-3$ & 170 \\
$2^{20}$ & $4.78 \mathrm{e}-6$ & 57.6 & $3.18 \mathrm{e}-2$ & 58.7 & $2.34 \mathrm{e}-4$ & 861 \\
\hline
\end{tabular}

Table XII

ALGORITHM COMPARISON OF THE RE FOR THE 100-DIMENSIONAL INTEGRAL (11)

\begin{tabular}{|c|c|c|c|}
\hline $\mathrm{T}$ & OP & FI & SO \\
\hline 1 & $2.67 \mathrm{e}-3$ & $7.18 \mathrm{e}-2$ & $9.31 \mathrm{e}-2$ \\
2 & $1.89 \mathrm{e}-4$ & $6.02 \mathrm{e}-2$ & $8.66 \mathrm{e}-2$ \\
10 & $3.22 \mathrm{e}-5$ & $4.12 \mathrm{e}-2$ & $6.94 \mathrm{e}-2$ \\
100 & $8.16 \mathrm{e}-7$ & $1.13 \mathrm{e}-2$ & $3.88 \mathrm{e}-3$ \\
\hline
\end{tabular}

Generalized Fibonacci numbers of the correspond-ing dimensionality, the best relative error is produced by the optimized lattice algorithm OPT - see Table I, but for a preliminary given time in seconds the optimized method OPT and the Fibonacci latice rule FIBO gives results of the same order - see Table II. For the 5-dimesnional integral again the best approach is OPT method, for $N=440096$ it gives relative error of $8.16 e-7$ see Table III, while for $20 s$ again FIBO method gives results of the same order as the optimized method - see Table IV. For the 8-dimensional integral the Adaptive approach, the Sobol QMC algorithm and the Fibonacci approach produce relative error of the same order - see Table V, but for a preliminary given time in seconds, Fibonacci approach is better than both Sobol QMC and Adaptive approach - see Table VI. For the 20dimensional integral Sobol QMC approach is better than both Fibonacci and Adaptive approach - see Table VII and Adaptive approach requires very huge amount of time - near one hour for number of samples $N=524888$ due to the division of the subareas in the description of the algorithm. Thats why we omit this algorithm for the 50 and 100-dimensional integrals. For $20 s$ for 20-dimensional integral the best result is produced again by the optimized lattice rule $-1.23 e-8$ in Table VIII. For the 50-dimensional integral Fibonacci approach is worse than Sobol approach by at least 1 order - see Table IX, but for a preliminary given time in seconds Sobol QMC and Fibonacci approach give relative errors of the same order - see Table $\mathrm{X}$. It is worth mentioning that the SOBOL approach requires more amount of time due to generation of the sequence, while Fibonacci lattice rules and Optimized approach are more faster and computationally efficient algorithms. For the 100-dimensional integral the best result is produced by the optimized lattice approach - it gives $4.78 e-6$ for number of samples $N=2^{20}$ - see Table XI and for $100 s$ it produces a relative error of $8.16 e-7$ which is very high accuracy and with 3 to 4 orders better than the other stochastic approaches. So we demonstrate here the advantages of the new lattice method and its capability to achieve very high accuracy for less than a minute on a laptop with a quad core CPU.

\section{CONCLUSION}

A comprehensive experimental study of optimized lattice rule, Fibonacci lattice sets, Sobol sequence and Adaptive approach has been done for the first time on some case test functions related to option pricing. Optimized lattice rule described here is not only one of the best available algorithms for high dimensional integrals but also one of the few possible methods, because in this work we show that the deterministic algorithms need a huge amount of time for the evaluation of the multidimensional integral, as it was discussed in this paper. The numerical tests show that the improved lattice rule is efficient for multidimensional integration and especially for computing multidimensional integrals of a very high dimensions up to 100. The novelty is that the new proposed optimized method gives very high accuracy for less than a minute on laptop even for 100-dimensional integral. It is an important element since this may be crucial in order to achieve a more reliable interpretation of the results in European style options which is foundational in computational finance.

\section{REFERENCES}

[1] N. Bakhvalov (2015) On the approximate calculation of multiple integrals, Journal of Complexity 31(4), 502-516.

[2] P.P. Boyle, Y. Lai and K. Tan, Using lattice rules to value lowdimensional derivative contracts (2001).

[3] Centeno, V., Georgiev, I. R., Mihova, V., \& Pavlov, V. (2019, October) Price forecasting and risk portfolio optimization. In AIP Conference Proceedings (Vol. 2164, No. 1, p. 060006). AIP Publishing LLC.

[4] Dimov I., Monte Carlo Methods for Applied Scientists, New Jersey, London, Singapore, World Scientific, 2008, 291p.

[5] Hua, L.K. and Wang, Y., Applications of Number Theory to Numerical analysis, 1981.

[6] L. K. Hua and Y. Wang, Applications of number theory to numerical analysis, (Springer 1981).

[7] F.Y. Kuo and D. Nuyens (2016) Application of quasi-Monte Carlo methods to elliptic PDEs with random diffusion coefficients - a survey of analysis and implementation, Foundations of Computational Mathematics 16(6), 1631-1696.

[8] Y. Lai and J. Spanier, Applications of Monte Carlo/Quasi-Monte Carlo methods in finance: option pricing, Proceedings of the Claremont Graduate University conference (1998).

[9] S.H. Paskov, Computing high dimensional integrals with applications to finance, Technical report CUCS-023-94, Columbia University (1994).

[10] I.H. Sloan and P.J. Kachoyan (1987) Lattice methods for multiple integration: Theory, error analysis and examples, SIAM J. Numer. Anal. 24, 116-128.

[11] I.H. Sloan and S. Joe, Lattice Methods for Multiple Integration, Lattice methods for multiple Integration, (Oxford University Press 1994).

[12] S. L. Zaharieva, I. Radoslavov Georgiev, V. A. Mutkov and Y. Branimirov Neikov, "Arima Approach For Forecasting Temperature In A Residential Premises Part 2," 2021 20th International Symposium infoteh-jahorina (Infoteh), 2021, pp. 1-5.

[13] Y. Wang and F. J. Hickernell (2000) An historical overview of lattice point sets, in MC and QMC Methods 2000, Proceedings of a Conference held at Hong Kong Baptist University, China. 